\title{
Genome Sequence Resource of Botryosphaeria dothidea CK16, a Fungal Pathogen Causing Chinese Hickory Trunk Canker Disease
}

\author{
Yuxin Rao, ${ }^{1}$ Li Mei, ${ }^{2}$ Liqin Zhang, ${ }^{3}$ Hong Jiang, ${ }^{1}$ Liangjin Ma, ${ }^{1}$ and Yongjun Wang ${ }^{1,2, \dagger}$ \\ ${ }^{1}$ College of Forestry and Biotechnology, Zhejiang A\&F University, Hangzhou, 311300, China \\ ${ }^{2}$ State Key Laboratory of Subtropical Silviculture, Zhejiang A\&F University, Hangzhou, 311300, China \\ ${ }^{3}$ Key Laboratory of Vector Biology and Pathogen Control of Zhejiang Province, Huzhou University, \\ Huzhou 313000, China
}

\begin{abstract}
Botryosphaeria dothidea is a latent fungal pathogen that causes cankers or diebacks on a variety of host woody plants worldwide. The symptomatic necrosis on the host plants can be triggered by abiotic stress, such as drought and soil acidification. Here we report a highquality genome assembly and announcement of the $B$. dothidea strain CK16 (CGMCC 19654), which causes trunk canker disease on Carya cathayensis in China. The genome sequence of strain CK16 will be useful for studying the evolution, host adaption, and pathogenicity of $B$. dothidea, which will be beneficial for a better understanding of the mechanisms of host-pathogen interaction during the endophytic period.
\end{abstract}

\section{Genome Announcement}

Botryosphaeria dothidea is a global pathogen causing cankers or diebacks on a variety of host woody plants (Marsberg et al. 2017). This pathogenic fungus interacts with host plants during a latent or endophytic phase (Slippers and Wingfield 2007). Infections of $B$. dothidea become symptomatic only under the conditions of host stresses, such as drought, physical damage, soil acidification, waterlogging, or other unsuitable growing environments (Marsberg et al. 2017). The endophytic period of up to 6 years on host trees makes it difficult to detect or control once established in the orchards (Marsberg et al. 2017). Conventionally, Botryosphaeria canker or dieback on woody plants is controlled through repeated fungicide applications (Moral et al. 2019). Trunk canker on Chinese hickory (Carya cathayens Sarg.) caused by $B$. dothidea is a yield-devastating disease in China, resulting in huge losses in nut production (Yao et al. 2019).

In this study, we present a high-quality draft genome sequence of a strain CK 16 derived from a single conidium culture of $B$. dothidea isolated from the canker tissue of $C$. cathayens in 2016 (Xu et al. 2018). The strain is currently deposited in the China General Microbiological Culture Collection Center (CGMCC 19654). The fungal genomic DNA was extracted and sequenced at the Hangzhou Keruidi Technology Co. Ltd. using Illumina MiSeq paired-end sequencing technology. In total, 146,162 paired reads of 300 bp (2.19 Gbp; average nuclear coverage 42x) were assembled using SPAdes v3.13.1 (Bankevich et al. 2012). The nuclear genome of $B$. dothidea consists of 1,303 scaffolds with a total assembly length of $44.39 \mathrm{Mbp}$ (N50 = 354,846 bp), 54.60\% GC-content, and a maximum scaffold size of 1,483,403 bp (Table 1). Genome completeness was assessed through the presence of conserved single-copy fungal genes using BUSCO v3 (Waterhouse et al. 2018). We used the fungi_odb9 data set,

${ }^{\dagger}$ Corresponding author: Y. J. Wang; wangyj@zafu.edu.cn

Y. X. Rao and L. Mei contributed to this work equally.

The author(s) declare no conflict of interest.

Accepted for publication 21 March 2021.
Funding

This research was supported by a grant from Zhejiang Key Research and Development Program of China (2019C0203002).

\section{Keywords}

Botryosphaeria dothidea, trunk canker disease, Chinese hickory 
Table 1. Genome features of Botryosphaeria dothidea CK16

\begin{tabular}{ll} 
Variables & Statistics \\
Sequence coverage & $42 \times$ \\
Assembly size & $44,391,467 \mathrm{bp}$ \\
Number of contigs & 1,303 \\
$\mathrm{~N}_{50}$ & $354,846 \mathrm{bp}$ \\
$\mathrm{GC}$ content & $54.60 \%$ \\
Number of coding genes & 13,856 \\
BUSCO completeness & $99.31 \%$ \\
\hline
\end{tabular}

identifying 293 of 321 (91.3\%) of these genes as present in the final assembly (Waterhouse et al. 2018). The nuclear genome was annotated using the MAKER2 pipeline (Holt and Yandell 2011). The Augustus 2.7 program was used to retrieve the related protein-coding genes for this fungus. In total, 13,856 protein-coding genes were identified with a total length of 38.69 Mbp, constituting $87.2 \%$ of the total genome. Functional annotation showed that 9,863 $(71.2 \%)$ genes were assigned with GO terms, most of which were involved in metabolic process $(32.5 \%)$, catalytic activity $(26.7 \%)$, cell process $(23.0 \%)$, binding $(7.7 \%)$, and response to stress or stimulus (5.5\%). Analysis with SignalP v5.0 (Almagro Armenteros et al. 2019) revealed that 176 predicted secreted proteins containing a secretion signal peptide.

The ability to degrade complex plant carbohydrates is an important aspect of the lifestyles of plant-associated fungi (Kubicek et al. 2014). B. dothidea is particularly well equipped with genes encoding carbohydrate-active enzymes (CAZymes). In total, 538 putative CAZymes were announced, of which 126 potential CAZymes were considered to be involved in pathogenicity based on the best-matched proteins from the database of pathogen-host interactions (http://www.phi-base.org/). The CAZyme categories contained 83 auxiliary activities (AAs) including 21 AA1 with degrading lactase activity, 136 carbohydrate binding modules (CBMs), 42 carbohydrate esterases (CEs), 186 glycoside hydrolases (GHs), 68 glycosyl transferases (GTs), and 23 polysaccharide lyases (PLs).

Repetitive sequences and transposable elements (TEs) increase the genome size and play vital roles in the genetic evolution and classification of fungal species (Muszewska et al. 2017). A total of $4,859,837 \mathrm{bp}$ (accounting for $10.9 \%$ of the genome) of repetitive sequences were identified in the $B$. dothidea CK16 isolate genome, including DNA transposons (35.4\%), tandem repeat (TRs) sequences (20.6\%), long terminal repeats (LTR) $(16.4 \%)$, short interspersed elements (SINE) (9.5\%), and other unclassified transposons (18.1\%) by using four types of prediction software.

One of the crucial weapons that necrotrophic, polyphagous pathogens possess is the production of phytotoxic compounds to kill cells of a range of plant species (Pusztahelyi et al. 2015). To identify the pathways involved in the production of secondary metabolites in $B$. dothidea, we searched the genomes for genes encoding key enzymes such as NRPS (non-ribosomal peptide synthetase), PKS (polyketide synthase), hybrid (PKS-NRPS), and DMATS (dimethylallyl tryptophane synthase). The strain was found to contain 46 genes encoding key secondary metabolism (SMs) biosynthesis enzymes. Compared with fungi with different lifestyles, these numbers are lower than for the hemibiotrophic model plant pathogen Colletotrichum spp. (Baroncelli et al. 2018; Gan et al. 2019; Liang et al. 2020).

In the present study, we provide the draft genome sequence of $B$. dothidea CK16, a strain causing trunk canker of $C$. cathayens in China. The sequence represents a new useful source for future research in the pathogenicity of $B$. dothidea on woody plants. The genome sequence described in this article has been deposited in GenBank under the accession number JACTUD000000000 (BioProject: PRJNA660898; BioSample: SAMN15963239).

\section{Literature Cited}

Almagro Armenteros, J. J., Tsirigos, K. D., Sønderby, C. K., Petersen, T. N., Winther, O., Brunak, S., von Heijne, G., and Nielsen, H. 2019. SignalP 5.0 improves signal peptide predictions using deep neural networks. Nat. Biotechnol. 37: 420-423.
Bankevich, A., Nurk, S., Antipov, D., Gurevich, A. A., Dvorkin, M., Kulikov, A. S., Lesin, V. M., Nikolenko, S. I., Pham, S., Prijbelski, A. D., Pyshkin, A. V., Sirotkin, A. V., Vyahhi, N., Tesler, G., Alekseyev, M. A., and Pevzner, P. A. 2012. SPAdes: a new genome assembly algorithm and its applications to single-cell sequencing. J. Comput. Biol. 19:455-477. 
Baroncelli, R., Sukno, S. A., Sarrocco, S., Cafà, G., Le Floch, G., and Thon, M. R. 2018. Whole-genome sequence of the orchid anthracnose pathogen Colletotrichum orchidophilum. Mol. Plant-Microbe Interact. 31:979-981.

Gan, P., Tsushima, A., Narusaka, M., Narusaka, Y., Takano, Y., Kubo, Y., and Shirasu, K. 2019. Genome sequence resources for four phytopathogenic fungi from the Colletotrichum orbiculare species complex. Mol. Plant-Microbe Interact. 32:1088-1090.

Holt, C., and Yandell, M. 2011. MAKER2: an annotation pipeline and genome database management tool for second generation genome projects. BMC Bioinformatics 12 : 491.

Kubicek, C. P., Starr, T. L., and Glass, N. L. 2014. Plant cell wall-degrading enzymes and their secretion in plant-pathogenic fungi. Annu. Rev. Phytopathol. 52:427-451.

Liang, X., Cao, M., Li, S., Kong, Y., Rollins, J. A., Zhang, R., and Sun, G. 2020. Highly contiguous genome resource of Colletotrichum fructicola generated using long-read sequencing. Mol. Plant-Microbe Interact. 33:790-793.

Marsberg, A., Kemler, M., Jami, F., Nagel, J. H., Postma-Smidt, A., Naidoo, S., Wingfield, M. J., Crous, P., Spatafora, J. W., Hesse, C. N., Robbertse, B., and Slippers, B. 2017. Botryosphaeria dothidea: a latent pathogen of global importance to woody plant health. Mol. Plant Pathol. 18:477-488.
Moral, J., Morgan, D., and Michailides, T. J. 2019. Management of Botryosphaeria canker and blight diseases of temperate zone nut crops. Crop Prot. 126:104927.

Muszewska, A., Steczkiewicz, K., Stepniewska-Dziubinska, M., and Ginalski, K. 2017. Cut-and-paste transposons in fungi with diverse lifestyles. Genome Biol. Evol. 9: 3463-3477.

Pusztahelyi, T., Holb, I. J., and Pócsi, I. 2015. Secondary metabolites in fungus-plant interactions. Front. Plant Sci. 6:573.

Slippers, B., and Wingfield, M. 2007. Botryosphaeriaceae as endophytes and latent pathogens of woody plants: diversity, ecology and impact. Fungal Biol. Rev. 21:90106.

Waterhouse, R. M., Seppey, M., Simão, F. A., Manni, M., loannidis, P., Klioutchnikov, G., Kriventseva, E. V., and Zdobnov, E. M. 2018. BUSCO applications from quality assessments to gene prediction and phylogenomics. Mol. Biol. Evol. 35:543-548.

Xu, S., Yao, J., Wu, F., Mei, L., and Wang, Y. 2018. Evaluation of Paenibacillus polymyxa carboxymethylcellulose/poly (vinyl alcohol) formulation for control of Carya cathayensis canker caused by Botryosphaeria dothidea. For. Pathol. 48:e12464.

Yao, J., Mei, L., Jiang, H., Hu, G., and Wang, Y. 2019. Evaluation of Carya cathayensis resistance to Botryosphaeria trunk canker using grafting on pecan. Sci. Hortic (Amsterdam) 248:184-188. 